\title{
A Modified Heuristic Approach of Logical Topology Design in WDM Optical Networks
}

\author{
Ebrahim E. Bedeer ${ }^{1}$, Hossam M. H. Shalaby ${ }^{2}$, El-Sayed A. El-Badawy ${ }^{3}$, Salah A. Khamis ${ }^{1}$ \\ ${ }^{1}$ The Electronics and Electrical Communication Eng. Dept, Fac. of Eng., Tanta University, \\ Egypt \\ ${ }^{2}$ The Electrical Eng. Dept, Fac. of Eng., University of Alexandria, Egypt \\ ${ }^{3}$ The Electronics and Communication Eng. Dept, Alexandria Higher Inst. of Eng. \& Technology, \\ Egypt \\ e.bedeer@ieee.org,shalaby@ieee.org,sbadawy@ieee.org,s.khamis@yahoo.com
}

\begin{abstract}
This paper introduces a modified heuristic approach as a solution to the problem of routing, wavelength assignment (RWA), and traffic grooming when designing a logical topology given the network physical topology. The RWA problem is known to be the assigning of network resources (wavelengths, transmitters, and receivers) to successfully establish lightpaths in the Wavelength Division Multiplexing (WDM) optical network. The traffic grooming problem is the multiplexing of lower speed connection requests to high speed streams. The proposed heuristic is based on Dijkstra's algorithm and it is a modification to the MSHT heuristic. For a given network physical topology and traffic pattern (traffic distribution between every node pairs), the objective is to design a logical topology and a routing algorithm so as to minimize both the average packet hop distance and amount of processing at each node. Two constraints are considered, namely, the number of wavelengths required to embed the resulting logical topology and the number of transmitters and receivers per node.
\end{abstract}

\section{INTRODUCTION}

The world today depends on information and knowledge much more than at any other time before. Most people have access to internet via high speed internet lines, and the number is increasing every day. $3.5 \mathrm{G}$ cell phones require more and more bandwidth to fulfill its new features. This means more bandwidth is needed every day. The solution to this problem either to install more fibers which is difficult and non economical solution, or to use WDM optical networks.

Wavelength Division Multiplexing (WDM) is a technology that multiplexes multiple optical carrier signals on a single optical fiber by using different wavelengths of laser light to carry different signals. It is used to compensate for the huge wasted fiber bandwidth, 50 Tera Hertz [5, 7], due to the limited speed of electronic devices at fiber ends that is limited to a few Giga bits per second [5, 7], with each carrier signal assigned to a certain wavelength. Current WDM optical networks have up to hundreds of wavelengths per fiber, 160 channel per fiber is practical today [7], with each wavelength operating at a bit rate of 2.5 Gbps or 10 Gbps [2].

The aim of this paper is to introduce a backbone WDM optical network for Lower Egypt. Using the physical topology of the current fiber network covering it, and based on a real value of traffic between main cities, we develop a heuristic approach that is based on Dijkstra's algorithm and considered as a modification to the MSHT heuristic presented in [3] in order to solve the network problem (routing, wavelength assignment, and traffic grooming).

The problem of logical topology design, routing, wavelength assignment (RWA), and traffic grooming is shown to be a MILP (mixed integer linear program) and classified as a complete NP-hard problem [1 - 7], which means it is difficult to solve for large size networks due to the limitation on LP solvers. Also it cannot be applied in real time due to the large processing time needed, so heuristics is used to give an approximate solutions with comparable results to the exact solution obtained from the MILP solution but in much less time which make it suitable to be applied for large network sizes and in real time.

A general structure of a network is shown in Fig. 1; an end node is either the source or the termination of a lightpath while a routing node is the intermediate node that a lightpath traverses between two end nodes. A 


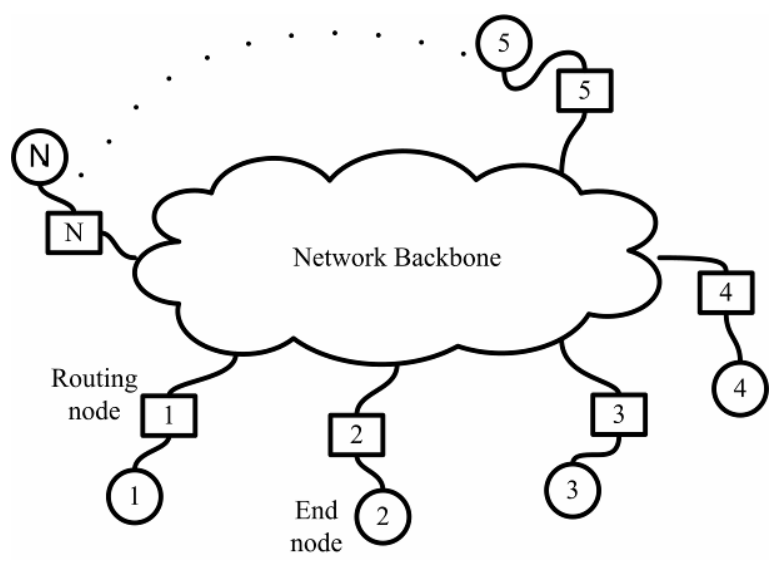

Fig. 1. Network structure

lightpath [1] is an all-optical path between its source and destination that must be on the same wavelength on all the fiber links that it transverses and without electronic conversion at intermediate nodes.

Generally it is not possible to establish direct lightpaths between every node pairs due to limited number of wavelengths per fiber and limited number of transceivers per node. Direct lightpaths are lightpaths that exist between the source node and the destination node without any electronic conversion hence there will be some node pairs that have to communicate via intermediate nodes. So the incoming lightpath to a node have to be converted from a given wavelength to another one so there is a need for optical WRS (wavelength routing switches) or wavelength converters at each node.

The physical topology of a network is the physical set of routing and end nodes, and the fiber links connecting them, while, the logical topology is the interconnection of nodes via lightpaths to carry network traffic. The physical degree of a node is the number of other nodes that is directly connected to it by fiber links, while, the logical degree of a node is the number of lightpaths originating from a node (logical our-degree) or the number of lightpaths terminating at a node(logical in-degree). In our work, the logical outdegree and the logical in-degree are the same, and we would point to them as the logical degree of a node. For more information about wide-area optical networks refer to $[5,6,7]$.

Section II describes the MILP optimization problem. Section III presents the proposed heuristic. Section IV gives a numerical example on Lower Egypt backbone network. Section V compare between our proposed heuristic and the MSHT heuristic. Section VI concludes the paper.

\section{PROBLEM DESCRIPTION}

The complete network model describing the network problem as an optimization problem can be found in $[3,7]$. It is omitted here to save space.

Objective function:

- Minimize: $\frac{1}{\sum_{s, d} \Lambda_{s d}} \sum_{i, j} \sum_{s, d} \lambda_{i j}^{s d}$

Where $\lambda_{i j}^{s d}$ is the traffic flowing from node $\mathrm{s}$ to node $\mathrm{d}$ and using the lightpath originating from node $\mathrm{i}$ and terminating at node $\mathrm{j}$ as an intermediate virtual link. $\Lambda_{\text {sd }}$ is the traffic value between node s and node $d$. Complete explanations of the notation used can be found in $[3,7]$.

Objective function may be to minimize average packet hop distance $[1,5]$, the average packet delay [2], cost [3], or the network congestion [2] which is equivalent to maximizing the total carried traffic per link. The objective function chosen here is to minimize the average packet hop distance which is inversely proportional to the total network throughput $[1,5]$. Average packet hop distance is the number of lightpaths that a packet has to transverse on average.

\section{Proposed Heuristic}

Our heuristic approach is based on Dijkstra's algorithm [8], and it is a modification to the MSHT (Maximize Single-Hop Traffic) heuristic presented in [3]. We use the Dijkstra's algorithm to find the shortest path between every node pair in the network. Our cost function when selecting the shortest path is the number of hops i.e. we select the path with the least number of intermediate hops. If this path is not available due to constraint limitations (no free wavelength or no free transmitter or receiver at the source or destination nodes, respectively), we select the second shortest path if available then the third shortest path and so on.

Our modification to the MSHT is that we give priority to neighboring nodes rather than node pairs with highest value of traffic. The idea is to insure that there will not be a case in which neighboring nodes communicate through intermediate nodes. If this happens it will be misusing of network resources. Another benefit that these direct lightpath between neighboring nodes will be used to carry the groomed traffic and hence will ensure that even groomed traffic get its shortest path also.

The proposed heuristic is as follow:

1. Establish a lightpath between every neighbor node pair. Assign a certain wavelength $\lambda$ to this lightpath.

2. Sort the traffic distribution matrix $T=\left(t_{i j}\right)$ in a descending 
TABLE I

TRAFFIC MATRIX (MBPS)

\begin{tabular}{|c|c|c|c|c|c|c|c|c|c|c|}
\hline & $\mathrm{CA}$ & ALEX & TA & DMN & SHB & KFR & MAN & ZAG & BEN & DMT \\
\hline $\mathrm{CA}$ & 0.00 & 1252.31 & 799.60 & 816.53 & 599.26 & 211.48 & 989.35 & 970.17 & 715.31 & 189.92 \\
\hline ALEX & 1286.394 & 0.00 & 224.35 & 86.67 & 13.15 & 20.31 & 18.18 & 59.36 & 4.25 & 4.32 \\
\hline TA & 833.1712 & 192.28 & 0.00 & 20.20 & 21.61 & 55.45 & 23.30 & 29.27 & 9.45 & 2.26 \\
\hline $\mathrm{DMN}$ & 840.2976 & 96.88 & 12.62 & 0.00 & 1.73 & 3.27 & 0.74 & 62.46 & 0.33 & 0.12 \\
\hline SHB & 617.9168 & 13.15 & 24.08 & 1.73 & 0.00 & 0.61 & 0.54 & 2.76 & 1.33 & 0.15 \\
\hline KFR & 211.8416 & 22.81 & 54.68 & 2.44 & 0.38 & 0.00 & 1.34 & 8.72 & 0.10 & 0.09 \\
\hline MAN & 1019.263 & 19.96 & 15.35 & 0.58 & 0.54 & 2.18 & 0.00 & 9.16 & 0.57 & 5.38 \\
\hline ZAG & 994.4864 & 59.36 & 7.48 & 62.46 & 2.76 & 8.78 & 9.16 & 0.00 & 2.94 & 0.13 \\
\hline BEN & 710.1325 & 5.16 & 21.24 & 0.26 & 1.55 & 0.15 & 0.44 & 3.86 & 0.00 & 0.08 \\
\hline DMT & 217.5744 & 1.04 & 1.28 & 0.11 & 0.14 & 0.43 & 6.96 & 0.26 & 0.08 & 0.00 \\
\hline
\end{tabular}

order.

3. Select the source destination pairs $\left(i_{\max }, j_{\max }\right)$ with the largest traffic.

If all source-destination pairs with nonzero traffic are tried, go to 5

4. If the node $i_{\max }$ has a free transmitter and the node $j_{\max }$ has a free receiver,

- Find the path with the lowest number of hops between $i_{\max }$ and $j_{\max }$ in the physical topology.

- If there is more than one path having the same number of hops, select the one with the shortest propagation-delay on the physical topology.

- Check if there is an available wavelength on the selected path.

- If there is not, Pick another path, check for an available wavelength.

- If there is not any, go to $\mathbf{5}$.

- If there is an available wavelength then

- Create the lightpath $i_{\max }, j_{\max }$, assign it to the minimum available $\lambda$

- Check if the traffic between $i_{\max }, j_{\max }$ is larger than the channel capacity or not. Remaining traffic $T\left(i_{\max }^{\prime}, j_{\max }^{\prime}\right)=$ channel capacity $-T\left(i_{\max }, j_{\max }\right)$

- Resort the traffic matrix replacing the traffic between $i_{\max }$ and $j_{\max }$ with the value of remaining traffic $T\left(i_{\max }^{\prime}, j_{\max }^{\prime}\right)$ - Go to 3

- else

- Mark the traffic between the nodes $i_{\max }$ and $j_{\text {max }}$ as unrouted traffic.

- end

$$
\text { - Go to } 3
$$

else

- Mark the traffic between the nodes $i_{\max }$ and $j_{\max }$ as unrouted traffic.

- Go to 3 end

5. Sort the unrouted traffic matrix in a descending order

6. Select the source destination pairs $\left(i_{\max }, j_{\max }\right)$ with the largest traffic.

7. Find the path with the lowest number of hops between $i_{\max }$ and $j_{\max }$ in the physical topology.

If there is more than one path having the same number of hops, select the one with the shortest propagation-delay on the physical topology.

8. Check if there is enough capacity in the selected path to carry the unrouted traffic between $i_{\max }$ and $j_{\max }$.

If there is not any paths with enough capacity, block this traffic

9. Put the traffic $T\left(i_{\max }, j_{\max }\right)=0$

10. Go to $\mathbf{5}$ until finishing all the elements of the unrouted traffic matrix.

This heuristic approach solves the problem of routing, wavelength assignment, and traffic grooming assuming wavelength converters exist at all nodes. To explain this heuristic we may divide it into two phases. The first one establishes lightpaths between all neighboring node pairs, assign them a certain wavelength, and then it sorts the node pairs in a descending order of traffic value and establishes direct lightpaths between them as much as possible i.e. the first phase constitutes the logical topology. Due to limited network resources, this phase will block traffic from some node pairs with lower value of traffic (because they are at the bottom of the traffic order). The second phase takes this blocked traffic and tries to groom them with lightpaths between neighboring nodes. It will not be a problem as groomed traffic is of lower value traffic.

Queuing delay at intermediate nodes is neglected compared with propagation delay between the two communicating nodes $[2,4]$. Wavelengths per fiber are numbered from 1 to $\mathrm{W}$ and wavelength assignment is done by first-fit algorithm i.e. we use the least available wavelength and assign it to the lightpath.

\section{NumericAl RESUltS}

This section presents a numerical example of the network design, routing, wavelength assignment, and traffic grooming problem, using the 10-node Lower Egypt backbone network. Figure 2 is the physical network topology. The links joining the neighboring 


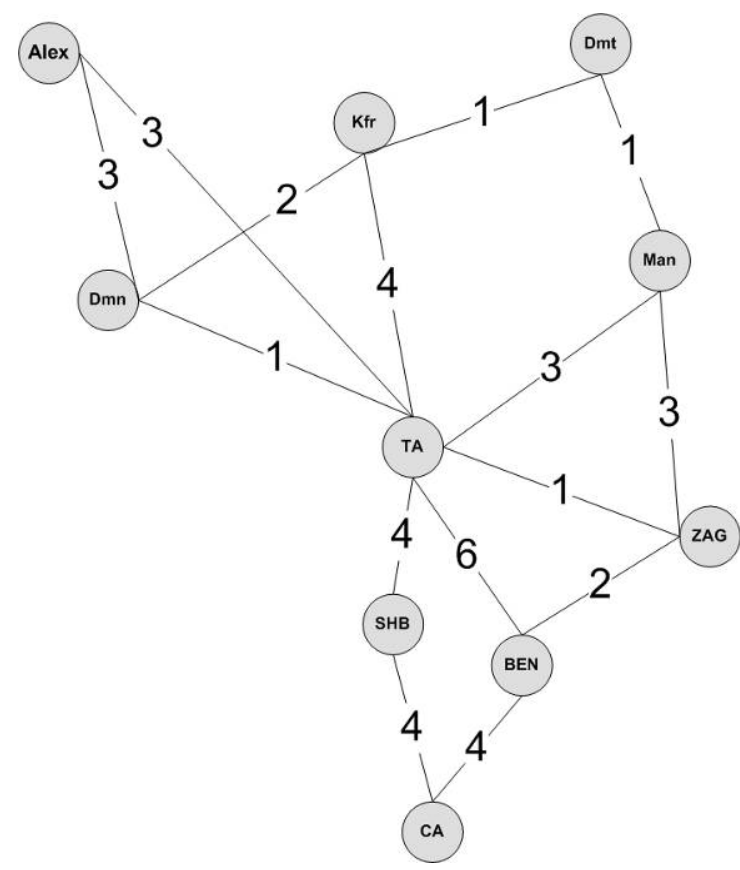

Fig. 2. 10-node Lower Egypt backbone.

nodes are bidirectional, i.e., there is a pair of unidirectional fibers that carries traffic in opposite directions. The numbers on the links represents the number of fiber cable installed between different cities. Our WDM network solution requires only one fiber cable and all remaining fiber cables may be used as a spare. Each node is equipped with an optical wavelength routing switch (WRS) that has complete wavelength conversion capabilities.

The traffic matrix, shown in Table 1, is the real value of traffic between Lower Egypt cities in Mbps, as given to us by Telecom. Egypt on June 2006. It includes voice traffic, cell phones traffic (only two operators), and internet traffic between various cities. You may notice that traffic values between nodes pairs differ greatly, and most of it is concentrated in Cairo. This is because both cell phone headquarters and internet service providers are located in Cairo.

Propagation delay between different cities is calculated by knowing the lengths of physical fiber links. We use the current channel capacity of the backbone fiber network used in Egypt, 2.5 Gbps,

Each node will be equipped with a number of fixed transmitters and receivers, with the node logical degree equals at least to the maximum physical degree of the same node [2]. You may notice that the physical degree of the backbone fiber network shown in Fig. 2 differs from 2, like CA node, to 7, like TA node, so our solution assumes different logical degree at each node and its value at each node starts from the physical

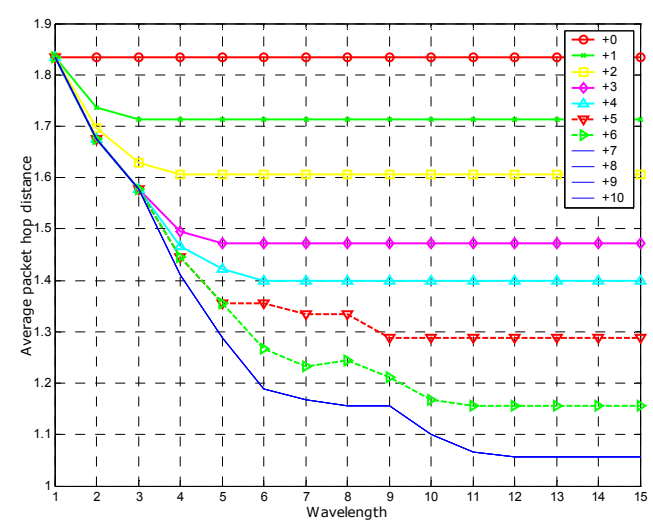

(a) Average packet hop distance vs. number of wavelengths per fiber.

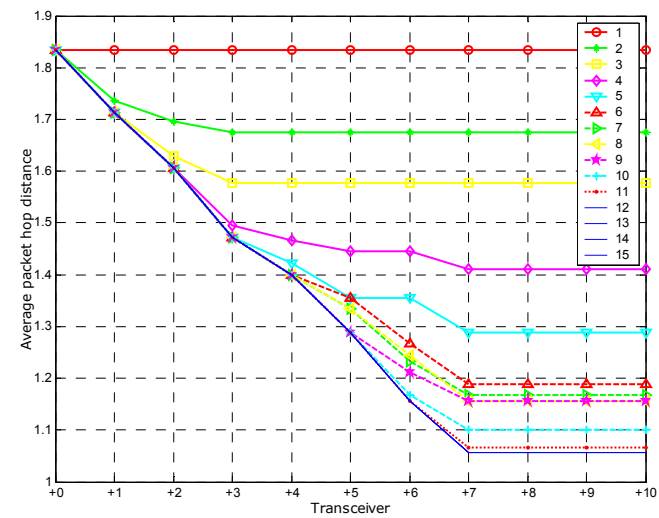

(b) Average packet hop distance vs. number of transceivers per node.

Fig. 3. Proposed heuristic results

degree at this node.

Figure 3(a) shows the average packet hop distance versus the number of wavelengths per fiber for different numbers of transceivers per node $(+0$ to +10$)$ $(+\mathrm{L}$ means that if the physical degree at a node is $\mathrm{M}$, then the logical degree at the same node is $\mathrm{M}+\mathrm{L}$ ). As shown in the figure, increasing the number of wavelengths per fiber reduces the average packet hop distance. This is logical and it was expected as increasing the number of wavelengths will increase the number of direct lightpaths between nodes (the more available wavelengths, the more the possibility that a lightpath continue traveling on the same wavelength without electronic conversion) and hence reduce the average packet hop distance. A further increase in the number of wavelengths will not improve the average packet hop distance and the curve will saturate, this is because the logical topology does not change and no more lightpaths are established as all the transceivers at all nodes are already used. For example, at +4 transceiver per node, we will not ever improve the 
average packet hop distance under 1.4 this is because no enough transceivers to make use of this huge number of wavelengths.

Figure 3(b) the average packet hop distance versus the number of transceivers per node for different values of wavelengths per fiber. Increasing the number of transceivers per node decreases the average packet hop distance (more direct lightpaths are established) until it saturates (logical topology does not change).

\section{COMPARISON BETWEEN Proposed HeUristiC AND MSHT HEURISTIC}

Figure 4 shows a comparison between the proposed heuristic and MSHT heuristic. Figure 4(a) plot the average packet hop distance versus the number of wavelengths per fiber at +9 transceivers per node, While Fig. 4(b) plots the average packet hop distance versus the number of transceiver per node at 7 wavelengths per fiber. It is clear that our heuristic performs better than modified MSHT heuristic, especially at fewer number of wavelengths per fiber (at 2 wavelengths per fiber, proposed heuristic gives 1.67 average packet hop distance versus 4.73 for modified MSHT). This can be explained as follow, for 2 wavelengths per fiber our proposed heuristic uses one wavelength for establishing direct lightpaths and uses the second wavelength for establishing lightpaths between neighbor nodes besides the groomed traffic. While the modified MSHT uses both wavelengths to carry direct ligthpaths besides groomed traffic.

The more the number of wavelengths per fiber, the more the number of direct lightpaths (as more network resources are available) and the less the number of groomed traffic, hence the two heuristics give nearly the same results as the number of wavelengths increase. At infinity of wavelengths per fiber both heuristics gives an average packet hop distance of 1 as there is enough network resources to establish alloptical lightpaths without grooming.

\section{CONCLUSION}

This paper presents a heuristic method to solve the logical topology design problem of routing and wavelength assignment in optical networks. We assume wavelength converters exist at all network nodes. The proposed solution should have a minimum of three wavelengths per fiber in order to carry all the traffic requests with a channel capacity of $2.5 \mathrm{Gbps}$ without blocking any connection requests.

The proposed heuristic gives better results than MSHT heuristic, especially, at lower number of wavelengths per fiber or lower number of transceivers per node.

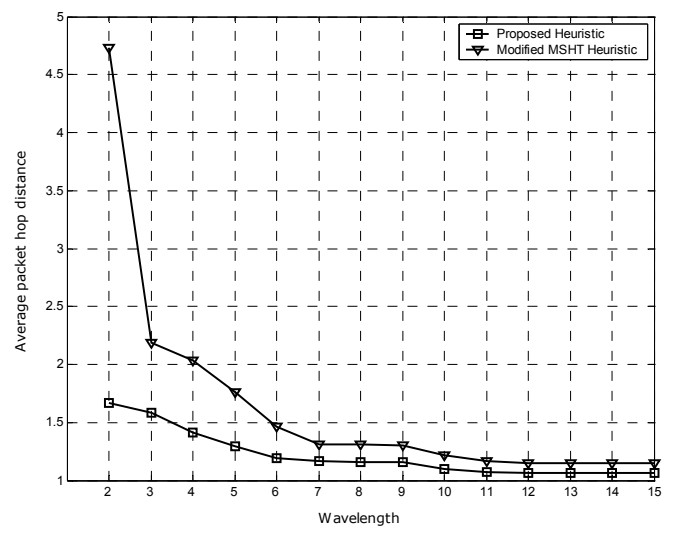

(a) Average packet hop distance vs. wavelength at 9 transceivers per node.

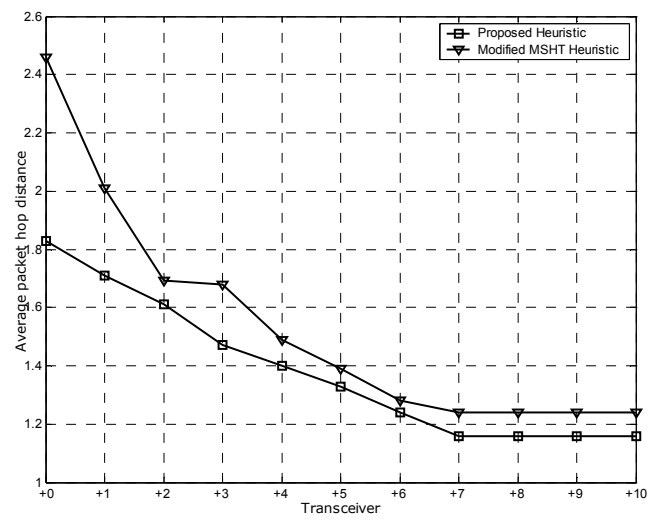

(b) Average packet hop distance vs. transceiver at 7 wavelengths per fiber.

Fig. 4. Comparison between proposed and MSHT heuristic

\section{REFERENCES}

[1] D. Banerjee and B. Mukherjee, "Wavelength-routed optical networks: linear formulation, resource budgeting tradeoffs, and a reconfiguration study," IEEE/ACM Trans. Networking, vol. 8, no. 5, Oct. 2000.

[2] R. Ramaswami, and K. N. Sivarajan. "Design of logical topologies for wavelength-routed optical networks," IEEE J, Select. Areas Commun., vol. 14, no. 5, pp. 840 - 851, June 1996.

[3] K. Zhu, H Zhu, and B. Mukherjee, Traffic Grooming in Optical WDM Mesh Networks. Springer 2005.

[4] H. Zang, J.P. Jue, L. Sahasrabuddhe, R. Ramamurthy, and B. Mukherjee, "Dynamic lightpath establishment in wavelength routed WDM networks," IEEE Commun. Mag., vol. 39, no. 9, pp. $100-108$, Sept. 2001 .

[5] B. Mukherjee, Optical Communication Networks. New York: McGraw Hill, 1997.

[6] B. Mukherjee, D. Banerjee, S. Ramamurthy, and A. Mukherjee, "Some principles for designing a wide-area optical network," IEEE/ACMTrans.Networking, vol. 4, pp. 684-696, Oct. 1996.

[7] B. Mukherjee. Optical WDM Networks. Springer, 2006.

[8] T. Cormen, C. Leiserson, R. Rivest, et al., Introduction to Algorithms. Cambridge: The MIT press, 1994. 